\title{
Nonlinear Signal Processing for Vocal Folds Damage Detection Based on Heterogeneous Sensor Network
}

\author{
Zhen Zhong \\ Baoju Zhang \\ Dept of Otolaryngology Head and Neck Surgery College of Electronic and Communication Engineering \\ Peking University First Hospital \\ Tianjin Normal University \\ Beijing 100034, China \\ Tianjin 300387, China \\ E-mail: zhong-zhen@sina.com \\ E-mail: wdxyzbj@163.com \\ Tariq S. Durrani \\ Shuifang Xiao \\ Dept of Electronic and Electrical Engineering \\ Dept of Otolaryngology Head and Neck Surgery \\ University of Strathclyde \\ Peking University First Hospital \\ Glasgow, Scotland, UK \\ Beijing 100034, China \\ E-mail: durrani@strath.ac.uk \\ E-mail: xiao-ent@163.com
}

\begin{abstract}
Heterogeneous sensor network-based medical decision making could facilitate the patient diagnosis process. In this paper, we present an intelligent approach for vocal folds damage detection based on patient's vowel voices using heterogeneous sensor network. Based on human voice samples and Hidden Markov Model, we show that transformed voice samples (linearly combined samples) follow Gaussian distribution, further we demonstrate that a type-2 fuzzy membership function (MF), i.e., a Gaussian MF with uncertain mean, is most appropriate to model the transformed voices samples, which motivates us to use a nonlinear signal processing technique, interval type-2 fuzzy
\end{abstract}

(C) 2015. This manuscript version is made available under the Elsevier user license http://www.elsevier.com/open-access/userlicense/1.0/ 
logic systems, to handle this problem. We also apply Short-Time-Fourier-Transform (STFT) and Singular-Value-Decomposition (SVD) to the vowel voice samples, and observe that the power decay rate could be used as an identifier in vocal folds damage detection. Two fuzzy classifiers, a Bayesian classifier, and a linear classifier are designed for vocal folds damage detection based on human vowel voices /a:/ and /i:/ only, and the fuzzy classifiers are compared against the Bayesian classifier and linear classifier. Simulation results show that an interval type-2 fuzzy classifier performs the best of the four classifiers.

Index Terms : Heterogeneous sensor network, interval type-2 fuzzy logic systems, Bayesian classifier, vocal folds, Short-Time-Fourier-Transform, singular-value decomposition.

\section{Introduction}

The proliferation of Heterogeneous Sensor Networks (HSN) has created a large amount of multi-sensor signals across multi-modality (e.g., optical, EO/IR, acoustic/seismic, RF, electromagnetic, mechanical, thermal, electrical, etc). Humans display a remarkable capability to perform multimodal signal processing despite noisy sensory signals and conflicting inputs. Humans are adept at network visualization, and at understanding subtle implications among the network connections. To date, however, human's innate ability to process and integrate signals from disparate, network-based sources has not translated well to signal processing in HSN. One of the great mysteries of the brain is cognitive control. How can the interactions between millions of neurons result in behavior that is coordinated and appears willful and voluntary? There is consensus that it depends on the prefrontal cortex (PFC) [1][2][3]. Many PFC areas receive converging inputs from at least two sensory modalities [4][5]. For example, the dorsolateral (DL) and ventrolateral PFC both receive projections from visual, auditory, 
and somatosensory cortex. Furthermore, the PFC is connected with other cortical regions that are themselves sites of multimodal convergence. The Stroop task, naming the color of a conflict stimulus (e.g., the word GREEN displayed in red) [6][8], and Wisconsin card sort task (WCST) [9] are variously described as tapping the cognitive functions of either selective attention, behavioral inhibition, top-down control, working memory, or rule-based or goaldirected behavior [1]. In this paper, we are interested in rule-based approach for decision making based on HSN. In [7], opportunistic sensing for HSN was studied for two different cases, with independent modalities and correlated modalities. It showed that HSN with independent modality sensors, we can make analysis and information fusion for each single modality sensor network. On decision making based on sensor networks, many interesting works have been reported, for example, target recongition [10][11][12], sense-through-wall human detection [13], threat assessment [15], resource allocation [16], localization [17], etc. In this paper, we are interested in an important medical decision making problem, vocal folds damage detection, based on heterogeneous sensor networks with optical and acoustic modalities.

In decision theory, ambiguity about probabilities should not affect choices. However, recent experiments [14] showed that many people are more willing to bet on risky outcomes (e.g., gambling on a roulette wheel) than on an ambiguous one (e.g., chance of a terrorist attack based on meager or conflicting evidence), holding the judged probability of outcomes constant. So, the confidence in judged probability can vary widely for "risky" and "ambiguous". Using functional brain imaging, Hsu et al [14] showed that the level of ambiguity in choices correlates positively with activation in the amygdala and orbitofrontal cortex, and negatively with a striatal system. This suggests that degree of uncertainty should be considered in decision making, contrary to traditional decision theory. So we introduce degree of uncertainty into vocal folds damage detection. The degree of uncertainty will be represented 
using type-2 fuzzy logic.

The presence of any form of damage to the delicate vocal fold cover tissue will impair phonation, be it a polyp, nodule, edema or scarring. Polyps of the vocal folds are a separate entity occurring nowhere else in the larynx or in the human body [18]. The pathological diagnosis of the vocal folds is a field which demands more investigation. Traditionally, the methods of diagnosis are indirect laryngoscope, video-laryngoscope, and stroboscope light [19]. However, most of these methods need special instrument, and mainly depend on the experience of the pathologists. In [20], measurement of vocal fold intraglottal pressure and impact stress were studied, and the experimental results match well with analytical predictions and support a current theory of mechanical trauma leading to vocal nodules. In [21], a nonlinear model was proposed to study chaotic vibrations of vocal folds with a unilateral vocal polyp.

Many engineering approaches for voice pathology detection have been reported. In [22], direct speech feature estimation using an iterative Expectation-Maximization (EM) algoritm was applied to vocal fold pathology detection. In [19], a measure of pitch perturbation was shown to be most useful in pathological discrimination. A robust, rapid and accurate system for automatic detection of normal and pathological speech was studied in [23], which employed non-invasive, non-expensive and fully automated measures of vocal tract characteristics and excitation information. In [24], jitter estimation over short time intervals (short-term jitter) was used for voice pathology detection in the case of running or continuous speech, where short-term jitter estimations were provided by the spectral jitter estimator, which was based on a mathematical description of the jitter phenomenon. In [25], a system for remotely detecting vocal fold pathologies using telephone-quality speech was presented, which used a linear classifier, processing measurements of pitch perturbation, amplitude perturbation and harmonic-to-noise ratio derived from digitized speech recordings. 
In [26], a neural network-based detector processing Mel frequency cepstral coefficients and their derivatives for normal/abnormal discrimination was presented. In [44], some preliminary results on vocal folds damage detection were presented based on patient voices. In [45], throat polyp detection based on compressed big data of voice with support vector machine algorithm was presented, and in [46] an intelligent throat polyp detection approach with separable compressive sensing was proposed.

In this paper, we are interested in detecting vocal folds damage using acoustic sensors based on the patient vowel voices only. Traditional pattern recognition techniques such as Bayesian classifier, known as the optimal classifier, could be used if the voice samples follow certain distribution, and this belongs to model-based statistical processing. As noted in [27], a shortcoming to model-based statistical signal processing is " $\ldots$ the assumed probability model, for which model-based statistical signal processing results will be good if the data agrees with the model, but may not be so good if the data does not." In human's voices, the voice amplitude is highly bursty, and we believe that no statistical model can really demonstrate the uncertain nature of the voice. Fuzzy logic systems (FLS) are model free. Their membership functions are not based on statistical distributions. In this paper, we, therefore, apply fuzzy techniques to vocal folds damage patient diagnosis.

In Section 2, we model voice samples using interval type-2 Gaussian membership function. In Section 3, Short-Time Fourier Transform and Singular-Value Decomposition are used to identify patient voice. Two fuzzy classifiers are presented in Section 4. In Section 5, a Bayesian classifier is proposed. Performances of four classifiers (including an existing linear classifier approach) are evaluated in Section 6. Conclusions are presented in Section 7. 


\section{Modeling Voice Samples Using Hidden Markov Model and Gaussian Primary MF with Uncertain Mean}

In [41], autoregressive Hidden Markov Model (HMM) was used to represent voice samples $x_{i}$, which means we could have

$$
x_{k}=-\sum_{i=1}^{p} b_{i} x_{k-i}+n_{k}
$$

where $n_{k}$ is Gaussian noise, and $b_{i}(i=1,2, \cdots, p)$ are the autoregression coefficients where $p$ is autoregressive order. So

$$
x_{k}-\sum_{i=1}^{p} c_{i} x_{k-i}=n_{k}
$$

where $c_{i}=-b_{i}$. Which means the difference between samples (or their linear combinations) follows Gaussian distribution.

The experiments were performed in the clinic of Department of Otolaryngology Head and Neck Surgery, Peking University First Hospital. In our experiment, the heterogeneous sensor network consists of acoustic and optical sensors. The optical sensors are indirect laryngoscope, which is used to visualize the patient's vocal folds by a means other than obtaining a direct line of sight. However, this approach is not very acurate because of the poor quality of optical information, and sometime the vocal folds damage is not visible because it's hidden. The acoustic sensors are embedded in laptop computers. A voice data collection software, Cool Edit Pro 2.0 was installed in a SONY laptop computer which was equipped with high quality microphone with single channel (acoustic sensor). Each patient kept a distance of $5 \mathrm{~cm}$ between mouth and microphone. Each patient was asked to pronounce/a:/ and/i:/ twice using conversational natural voice. The sampling rate was $192 \mathrm{KHz}$, and the data resolution was 32 bits. 20 patients participated in the test, of which 10 were male, and 10 were female. The patients were in ages of 28 to 81 , and 10 patients were 
normal, and 10 patients had vocal folds damage. Since the acoustic and optical modalities are independent in this experiment, we focus on acoustic modality in this paper.

Based on the voice data we have collected, we observed that the vowel /a:/ samples $\left(x_{k}\right)$ do not follow Gaussian distribution, as illustrated in Fig. 1a, but when we choose $p=5$, $c_{1}=c_{2}=c_{3}=c_{4}=0, c_{5}=1$, i.e.,

$$
x_{k}-x_{k-5}=n_{k}^{a}
$$

the new sequences follow Gaussian distribution, as illustrated in Fig. 1b. Similarly, we observed that the vowel /i:/ samples $\left(x_{k}\right)$ do not follow Gaussian distribution, as illustrated in Fig. 2a, but if we choose $c_{1}=1 p=1$, i.e.,

$$
x_{k}-x_{k-1}=n_{k}^{i}
$$

follows Gaussian distribution, as illustrated in Fig. 2.

We, therefore, tried to model the the new transformed voice sequences $n_{k}^{a}$ and $n_{k}^{i}$, to see if a Gaussian MF can match its nature. For $n_{k}^{a}$ and $n_{k}^{i}$ from each subject (human) for 100,000 samples, we equally separate it into 10 segments, and computed the mean $m_{i}$ and standard deviation (std) $\sigma_{i}$ of the $i$ th segment, $i=1,2, \cdots, 10$. We also computed the mean $m$ and std $\sigma$ of the entire sequence (100,000 samples). To see which value $-m_{i}$ or $\sigma_{i}$ - varies more, we normalized the mean and std of each segment using $m_{i} / m$, and $\sigma_{i} / \sigma$, and we then computed the std of their normalized values, $\sigma_{m}$ and $\sigma_{s t d}$. As we see from the last row of Tables $1, \sigma_{m} \gg \sigma_{s t d}$. We conclude, therefore, that if the transformed voice samples of each segment (short range) of the voice samples are Gaussian distributed, then the transformed voice samples in an entire voice samples (long range) are more appropriately modeled as a Gaussian with uncertain mean. This justifies the use of the Gaussian MFs with uncertain mean to model the transformed voice samples. 


\section{Identifying Vocal Folds Damage Patient Voice Using Short-Time Fourier Transform and Singular-Value} Decomposition

STFT uses a slide window to determine the sinusoidal frequency and phase content of a signal as it changes over time. This form of the Fourier transform, also known as timedependent fourier transform, has a great many applications in speech processing. We will show that STFT - based approach could be used as an identifier for vocal folds damage detection.

For the continuous-time signal, the function to be transformed is multiplied by a nonzero window sliding along the time axis, therefore a two-dimensional expression can be defined as:

$$
F(m, w)=\int_{-\infty}^{\infty} x(t) w(t-m) e^{-j w t} d t
$$

where $x(t)$ is the function to be transformed and $w(t)$ is the window function. $F(m, w)$ represents sinusoidal values at the center of the window $\mathrm{w}(\mathrm{t})$ that starts at time position $\mathrm{m}$. The discrete STFT can be expressed as

$$
F(m, w)=\sum_{n=0}^{N-1} x(n) w(n-m) e^{-j w n}
$$

We apply a rectangular window, with its length 2048 and step size 1024. In Fig. 3, we plot the spectrogram $\mid F(m, w \mid$ of a vocal folds damage patient vowel voices versus that of a normal person. Observe that the spectrogram could be used as an identifier in vocal folds damage detection.

The STFT of the voices is a matrix, how to extract its information for vocal folds damage detection? We use singular-value decomposition (SVD). The SVD is an important factorization of a rectangular real or complex matrix, with many applications in signal processing and statistics. Applications which employ the SVD include computing the pseudoinverse, 
least squares fitting of data, matrix approximation, and determining the rank, range and null space of a matrix. Given $P \in C^{N \times M}$ (assuming $N>M$ ), and $\operatorname{rank}(P)=r \leq M$. Determine a numerical estimate $r^{\prime}$ of the rank of the data sets matrix $P$ by calculating the singular value decomposition

$$
P=U\left[\begin{array}{ll}
\Sigma & 0 \\
0 & 0
\end{array}\right] V^{T},
$$

where, $U$ is an $N \times N$ matrix of orthonormalized eigenvectors of $P P^{T}, V$ is an $M \times$ $M$ matrix of orthonormalized eigenvectors of $P^{T} P$, and $\Sigma$ is the diagonal matrix $\Sigma=$ $\operatorname{diag}\left(\sigma_{1}, \sigma_{2}, \ldots, \sigma_{r}\right)$, where $\sigma_{i}$ denotes the $i^{t h}$ singular value of $P$, and $\sigma_{1} \geq \sigma_{2} \geq \cdots \geq \sigma_{r}>0$. Using SVD, the STFT of voices could be diagonalized, and the diagonal values in $\Sigma$ could be used to represent the speaker voice power decay in the frequency domain. Power decay rate means the how fast signal powers are reduced from one frequency to another. Generally the $\sigma_{1}$ is much higher than $\sigma_{2}$, and the decay from $\sigma_{1}$ to $\sigma_{2}$ somehow represent how a person could handle his voice freely. For illustration purpose, we plot the singular values $\left(\sigma_{i}\right)(i=1,2, \cdots, 10)$ in Fig. 4 for the two patients whose spectrogram were plotted in Fig. 3. Observe Fig. 3, the voice power decay rate, i.e., $P d=\sigma_{1}-\sigma_{2}$, is higher for a normal person than that of a patient with vocal folds damage, which means that a normal person could handle his/her voices more freely (with higher power changes from one frequency to another frequency). So voice power decay rate could be used as an identifier on vocal folds damage detection. In this paper, we will use the vowel /a:/ and /i:/ power decay rate in fuzzy classifiers for vocal folds damage detection. 


\section{Two Fuzzy Classifiers for Vocal Folds Damage De- tection}

In this Section, we use type-1 and interval type-2 FLSs for vocal folds damage detection.

\subsection{Type-1 FLSs}

A FLS is described by fuzzy IF-THEN rules which represent input-output relations of a system. For classification, in a type-1 FLS with a rule base of $M$ rules, each having $p$ antecedents, the $l$ th rule, $R^{l}$, is expressed as:

$$
R^{l} \text { : IF } x_{1} \text { is } \mathrm{F}_{1}^{l} \text { and } x_{2} \text { is } \mathrm{F}_{2}^{l} \text { and } \ldots \text { and } x_{p} \text { is } \mathrm{F}_{p}^{l} \text { THEN } y^{l}=c^{l}
$$

in which $l=1,2, \ldots, M$, the consequent $c^{l}$ are crisp values (e.g., +1 or -1 in this paper), and, $\mathrm{F}_{k}^{l}(k=1,2, \ldots, p)$ are type-1 fuzzy sets. For a singleton FLS, the rule firing degree is

$$
f^{l}=\mathcal{T}_{k=1}^{p} \mu_{\mathrm{F}_{k}^{l}}\left(x_{k}\right)
$$

where $\mathcal{T}$ denotes a $t$-norm (minimum or product).

In this paper, we use an unnormalized output [42] for the FLS, namely

$$
y=\sum_{l=1}^{M} f^{l} y^{l}
$$

because we make a decision based on the sign of the output, and normalization operation will not change the sign. If the domain of interest had 3 or more choices, we would use a normalized output for decision making.

In Section 6, we use type-1 FLSs for vocal folds damage detection based on voice samples only. 


\subsection{Interval Type-2 FLSs}

In a type- 2 FLS with a rule base of $M$ rules, in which each rule has $p$ antecedents, the $l$ th rule $R^{l}$, is expressed as:

$$
R \text { : IF } x_{1} \text { is } \tilde{\mathrm{F}}_{1}^{l} \text {, and } x_{2} \text { is } \tilde{\mathrm{F}}_{2}^{l} \text {, and, ..., and } x_{p} \text { is } \tilde{\mathrm{F}}_{p}^{l} \text { THEN } y^{l}=c^{l} \text {. }
$$

The firing degree, $F^{l}$, of rule $R^{l}$ is

$$
F^{l}=\sqcup_{\mathbf{x} \in \mathbf{X}}\left\{\left[\mu_{\tilde{\mathrm{X}}_{1}}\left(x_{1}\right) \sqcap \mu_{\tilde{\mathrm{F}}_{1}^{l}}\left(x_{1}\right)\right] \sqcap \cdots \sqcap\left[\mu_{\tilde{\mathrm{X}}_{p}}\left(x_{p}\right) \sqcap \mu_{\tilde{\mathrm{F}}_{p}^{l}}\left(x_{p}\right)\right]\right\}
$$

where $\mathbf{X}=X_{1} \times X_{2} \times \cdots \times X_{p}$ is the measurement domain of $\mathbf{x}=\left[x_{1}, x_{2}, \cdots, x_{p}\right]$, and $\sqcup$ and $\sqcap$ are join and meet operations [28]. General type-2 FLSs are computationally intensive because it is not easy to evaluate $F^{l}$ in (10). Things simplify a lot when secondary membership functions are interval sets, in which case secondary memberships are either 0 or 1 , such simplifications make the use of a type-2 FLS practical.

In [28], Liang and Mendel proposed the theory and design of interval type-2 fuzzy logic systems. They proposed an efficient and simplified method to compute the input and antecedent operations for interval type-2 FLSs, one that is based on a general inference formula for them. They introduced the concept of upper and lower membership functions (MFs) and illustrate their efficient inference method for the case of Gaussian primary MFs. They also proposed a method for designing an interval type-2 FLS in which they tuned its parameters [29]. They applied interval type-2 FLS to a number of very important applications where uncertainties abound, such as fading channel equalization [32] and co-channel interference elimination [33], network video traffic modeling and classification [34], connection admission control for ATM network [35]. Recently, interval type-2 FLSs were applied to sensor network lifetime estimation[36], and cross-layer optimization in ad hoc networks[37][38], event detection [39][40], etc. 
In Section 6, we use type-1 and interval type-2 FLSs as fuzzy classifiers for vocal folds damage detection. Our fuzzy classifiers are model-free. To compare them against a modelbased classifier, we develop a Bayesian classifier in Section 5.

\section{Bayesian Classifier for Vocal Folds Damage Detec- tion}

Bayesian decision theory [43] provides the optimal solution to the general decision-making problem. We assume that each patient has equal probability to have vocal folds damage, i.e., $H_{1}$ : Damaged, and $H_{2}$ : Normal, so $p\left(H_{1}\right)=p\left(H_{2}\right)=0.5$. If each transformed vowel voice samples (/a:/ and /i:/) of patient $j$ follows Gaussian distribution, $\mathbf{x}_{j} \triangleq\left[x_{j}^{a} x_{j}^{i}\right]^{T}$ stands for the samples from patient $\mathrm{j}$ for vowel /a:/ and /i:/, then

$$
p\left(\mathbf{x}_{j} \mid v_{j}\right)=\frac{1}{(2 \pi)\left|\mathbf{\Sigma}_{\mathbf{j}}\right|^{1 / 2}} \exp \left[-\frac{1}{2}\left(\mathbf{x}_{j}-\mathbf{m}_{j}\right)^{T} \mathbf{\Sigma}_{\mathbf{j}}{ }^{-1}\left(\mathbf{x}_{j}-\mathbf{m}_{j}\right)\right]
$$

where $\mathbf{m}_{j} \triangleq\left[m_{j}^{a}, m_{j}^{i}\right]^{T}$ and $\boldsymbol{\Sigma}_{\mathbf{j}}=\operatorname{diag}\left\{\sigma_{j}^{a 2}, \sigma_{j}^{i^{2}}\right\}$ are the mean vector $(2 \times 1)$ and covariance matrix $(2 \times 2)$ of $\mathbf{x}_{j}$. In this case,

$$
\begin{aligned}
& p\left(\mathbf{x} \mid H_{1}\right)=\sum_{i=1}^{10} p\left(\mathbf{x} \mid v_{i}\right) p\left(v_{i}\right) \\
& p\left(\mathbf{x} \mid H_{2}\right)=\sum_{i=11}^{20} p\left(\mathbf{x} \mid v_{i}\right) p\left(v_{i}\right)
\end{aligned}
$$

Based on Bayes decision theory, since $p\left(H_{1}\right)=p\left(H_{2}\right)=0.5$, we obtain the decision rule:

$$
\begin{array}{rr}
\text { Claim vocal folds damage if } & p\left(\mathbf{x} \mid H_{1}\right)>p\left(\mathbf{x} \mid H_{2}\right) \\
\text { No vocal folds damage if } & p\left(\mathbf{x} \mid H_{1}\right)<p\left(\mathbf{x} \mid H_{2}\right) \\
\text { Not sure if } & p\left(\mathbf{x} \mid H_{1}\right)=p\left(\mathbf{x} \mid H_{2}\right)
\end{array}
$$

This Bayesian vocal folds damage detector will be used in Section 6 . 


\section{Simulations}

We extract the general features and behavior of /a:/ and /i:/ voices for 20 patients, of which 10 have vocal folds damage and 10 have no vocal folds damage, and determine one discriminant rule for each patient in the domain of interest. In choosing the antecedents of the fuzzy classifier, we make full use of the statistical knowledge (mean and std) obtained from the patient voices. We used 100,000 samples in vowel /a:/ and /i:/ respectively to establish a discriminant rule for each patient. All-in-all, we obtained 20 rules, one per patient.

To evaluate the performance of the 2 fuzzy detectors, we used another group of 20 patients (testing group), which has no overlap with the first group of 20 patients whose vowel samples were used for fuzzy rules. By this means, it would help to demonstrate that our classifiers are robust. We also collected 100,000 voice samples for /a:/ and /i:/ respectively for each patient in the testing group. To demonstrate that our classifiers are able to detect vocal folds damage using a small number of samples, we made our detection based on every 5,000 samples, with 20 independent detections $(20 \times 5,000)$ for each patient. During testing, we obtain the mean $\mathbf{m}^{\mathbf{t}}=\left[m_{a}^{t}, m_{i}^{t}\right]$ for each 5,000 /a:/ and /i:/ samples.

\subsection{Design of Four Vocal Folds Damage Detectors}

\subsubsection{Design of Type-1 Fuzzy Vocal Folds Damage Detector}

For a type- 1 fuzzy classifier, the $l$ th rule, $R^{l}$, is $(l=1, \cdots, 10)$ :

$R^{l}$ : IF the transformed /a:/ voice is $\mathrm{F}_{1}^{l}$ and the transformed /i:/ voice is $\mathrm{F}_{2}^{l}$ and /a:/ power decay rate is $\mathrm{F}_{3}^{l}$ and /i:/ power decay rate is $\mathrm{F}_{4}^{l}$ THEN this patient has vocal folds damage $(+1)$ [or normal $(-1)]$.

The antecedents $\mathrm{F}_{k}^{l}(k=1,2,3,4)$ are described by a type-1 Gaussian MF whose mean, $m_{p}^{l}$, and std, $\sigma_{p}^{l}$, are determined by known patient voice samples. More specifically, $m_{1}^{l}$ and $\sigma_{1}^{l}$ 
are the mean and std of voice /a:/ samples in the 100,000 samples of patient $l$ in the first group; $m_{2}^{l}$ and $\sigma_{2}^{l}$ are the mean and std of /i:/ samples in the 100,000 samples of patient $l$ in the first group. To determine $m_{3}^{l}, \sigma_{3}^{l}, m_{4}^{l}$, and $\sigma_{4}^{l}$, we partition the voice samples into 10 segments for /a:/ and /i:/ respectively, and obtain the STFT of each segment. Then apply SVD to the STFT matrix to obtain the power decay rate for each segments. The mean and std of the 10 power decay rates are $m_{3}^{l}\left(m_{4}^{l}\right)$ and $\sigma_{3}^{l}\left(\sigma_{4}^{l}\right)$. The consequent $y^{l}=c^{l}$ in Section 4.1 corresponds to $y^{l}=+1$ (vocal folds damage) or $y^{l}=-1$ (normal) in the fuzzy detector. The +1 or -1 for $y^{l}$ is truely a representation of linguistic using numerical value. Since it's a binary decision, so we use +1 or -1 .

For a type-1 fuzzy detector, its input, $\mathbf{m}^{\mathbf{t}}=\left[m_{a}^{t}, m_{i}^{t}, P d_{a}, P d_{i}\right]$, is obtained from 5,000 vowel samples from patient in the testing group. $P d_{a}$ and $P d_{i}$ are the power decay rate for /a:/ and /i:/.

\subsubsection{Design of Type-2 Fuzzy Vocal Folds Damage Detector}

For type-2 fuzzy classifiers, the $l$ th rule, $R^{l}$, is $(l=1, \cdots, 10)$ :

$R^{l}$ : IF the transformed /a:/ voice is $\tilde{\mathrm{F}}_{1}^{l}$ and the transformed /i:/ voice is $\tilde{\mathrm{F}}_{2}^{l}$ and /a:/ power decay rate is $\mathrm{F}_{3}^{l}$ and /i:/ power decay rate is $\mathrm{F}_{4}^{l}$ THEN this patient has vocal folds damage $(+1)$ [or normal $(-1)]$.

The antecedents $\tilde{\mathrm{F}}_{k}^{l}(k=1,2,3)$ are described by a type-2 MF, i.e., a Gaussian MF with uncertain mean, whose mean $m_{k}^{l} \in\left[m_{k 1}^{l}, m_{k 2}^{l}\right]$ and std $\sigma_{k}^{l}$ are determined by the voice samples of patients in the first group. $\mathrm{F}_{3}^{l}$ and $\mathrm{F}_{4}^{l}$ are same as those in type- 1 fuzzy detector.

More specifically, $\sigma_{k}^{l}(k=1,2)$ are determined using the same method as described in Section 6.1.1, and $m_{k 1}^{l}$ and $m_{k 2}^{l}$ are determined as follows. We divided the 100,000 frames of the $l$ th known patient into 10 equal-length (10,000 samples) segments, and computed the 
mean $m_{1}^{l j}$ of $/$ a:/ samples in the $j$ th segment $(j=1, \cdots, 5)$. Let

$$
\begin{aligned}
& m_{11}^{l}=\min _{j=1, \cdots, 10} m_{1}^{l j} \\
& m_{12}^{l}=\max _{j=1, \cdots, 10} m_{1}^{l j}
\end{aligned}
$$

so $\left[m_{11}^{l}, m_{12}^{l}\right]$ is the range of uncertain mean of /a:/ voice samples of the $l$ th known patient. We obtained the ranges of uncertain mean of $/ \mathrm{i}$ :/ samples $\left(\left[m_{21}^{l}, m_{22}^{l}\right]\right)$ in a similar manner.

For a type-2 fuzzy detector, its input, $\left.\mathbf{m}^{\mathbf{t}}=\left[m_{a}^{t}, m_{i}^{t}, P d_{a}, P d_{i}\right]\right]$, is obtained from 5,000 vowel samples from patient in the testing group.

\subsubsection{Design of Bayesian Classifier}

Observe from (11), that the Bayesian classifier needs $\mathbf{m}_{i}=\left[m_{i}^{a}, m_{i}^{i}\right]^{T}$ and $\boldsymbol{\Sigma}_{\mathbf{i}}=\operatorname{diag}\left\{\sigma_{i}^{a 2}, \sigma_{i}^{i^{2}}\right\}$. In our design, $m_{i}^{a}$ and $\sigma_{i}^{a}$ are the mean and std of vowel /a:/ in the 100,000 samples of patient $i$ in the first group; similarly, $m_{i}^{i}$ and $\sigma_{i}^{i}$ are the mean and std of vowel $/ \mathrm{i}$ :/ in the 100,000

samples of patient $i$ in the first group; and, its input $\mathbf{x} \triangleq \mathbf{m}^{\mathbf{t}}$, where $\mathbf{m}^{\mathbf{t}}$ is obtained from the mean value of 5,000 voice samples from a patient in the testing group.

\subsubsection{Design of Linear Classifier}

We compared our approaches with an existing approach, linear classifier proposed in [25], which processing measurements of pitch perturbation, amplitude perturbation, and homonicto-noise ratio derived from digitized speech recording. The linear classifier can be summarized as follows. Let $\mathbf{x}$ be a column vector containing $d$ feature values which is assigned to one of two classes, i.e., normal or damaged. Based on the training sets, we could obtain the mean vectors $\mathbf{m}_{1}, \mathbf{m}_{2}$, and covariance matrix $\Sigma$ (for both classes) of the $\mathbf{x}$. Assume the prior probability of both classes are equal, the discriminant value $y$ is

$$
y=\left(\mathbf{m}_{1}-\mathbf{m}_{2}\right)^{T} \Sigma^{-1} \mathbf{x}-\frac{1}{2}\left(\mathbf{m}_{1}-\mathbf{m}_{2}\right)^{T} \Sigma^{-1}\left(\mathbf{m}_{1}-\mathbf{m}_{2}\right)
$$


and then the posterior probabilities for class 1 (normal) and class 2 (damaged) are then calculated using

$$
\begin{aligned}
& P(1 \mid \mathbf{x})=\frac{\exp (y)}{\exp (y)+1} \\
& P(2 \mid \mathbf{x})=1-P(1 \mid \mathbf{x})
\end{aligned}
$$

The final classification decision is obtained by choosing the class with the highest posterior probability.

In the pitch perturbation features, we used mean value of $F_{0}$, maximum $F_{0}$, minimum $F_{0}$, std of $F_{0}$, phonatory frequency range, mean absolute jitter, jitter, pitch perturbation quotient (smoothed over 5 pitch periods). In the amplitude perturbation features [25], we used mean, maximum, minimum, std, mean absolute shimmer, shimmer percentage, shimmer decibels, amplitude perturbation quotient smoothed over 5 pitch period. In Harmonic-to-Noise ratio is calculated based on the procedure given in [25]. The decision is made based on human vowel voices /a:/ or /i:/ only.

\subsection{Performance Analysis}

We computed the average probability of miss detection $\left(p_{r}(\epsilon)\right)$ for each fuzzy detector as well as for the Bayesian detector in $20 \times 20=400$ independent classifications (20 patients each with 20 5,000-sample segments), and please be aware that the voices of the first group patients were used to design the fuzzy rules, and the testing group of patients have no overlap with the first group. Simulations show that $p_{r}(\epsilon)=25 \%$ for Bayesian classifier, $p_{r}(\epsilon)=18 \%$ for type-1 fuzzy classifier, and $p_{r}(\epsilon)=14 \%$ for type- 2 fuzzy classifier. We evaluated the linear classifier in [25], and simulations show that $p_{r}(\epsilon)=21 \%$.

Bayesian classifier is known as the optimal classifier [43], but why it can't achieve the best performance? There are several possible reasons. 
1. Fuzzy classifiers use more identifiers, i.e., transformed voice samples as well as voice power decay rate from STFT and SVD, whereas Bayesian classifier only uses transformed voice samples.

2. The voice power decay rate from STFT and SVD doesn't follow any statistical distribution, so this knowledge couldn't be incorporated into Bayesian classifier.

3. The transformed voice samples do not exactly follow Gaussian distribution, but a Gaussian with uncertain means. Bayesian classifier is a model-based approach, so it can't achieve the best performance when the model doesn't match real-world data.

The linear classifier could not perform well because the training group data and the testing group data have no overlap, so the mean vector and variance matrix obtained from the training data couldn't fully reflect the pitch, amplitude, and harmonic-to-noise ratio in the testing data.

Although type-2 fuzzy classifier performs the best, it has highest complexity among the four classifiers. In Table 2, we summarize the classification performance and computational complexity of the four classifiers.

\section{Conclusions}

We have presented a new approach for vocal folds damage detection based on patient's vowel voices using interval type-2 fuzzy logic system. Based on human voice samples and Hidden Markov Model, we showed that transformed voice samples (linearly combined samples) follow Gaussian distribution, further we demonstrated that a type-2 fuzzy MF, i.e., a Gaussian MF with uncertain mean, is most appropriate to model the transformed voices samples. We also applied STFT and SVD to the vowel voice samples, and observe that the voice power decay 
rate could be used as an identifier in vocal folds damage detection. Two fuzzy classifiers and a Bayesian classifier were designed for vocal folds damage detection based on human vowel voices /a:/ and /i:/ only, and the fuzzy classifiers are compared against the Bayesian classifier and a linear classifier. Simulation results showed that an interval type-2 fuzzy classifier performs the best of the four classifiers.

\section{Acknowledgement}

This work was supported by The Royal Society of Edinburgh and National Science Foundation of China (NSFC) under Grant 61411130159.

\section{References}

[1] E. K. Miller, J. D. Cohen, "An Integrative Theory of Prefrontal cortex function," Annu. Rev. Neurosci., vol. 24, pp. 167, 2001.

[2] R. C. OReilly, "Biologically Based Computational Models of High-Level Cognition," Science, Oct 2006.

[3] Q. Liang, "Situation Understanding Based on Heterogeneous Sensor Networks and Human-Inspired Favor Weak Fuzzy Logic System," IEEE Systems Journal, vol. 5, no. 2, pp. 156-163, June 2011.

[4] D. A. Chavis, D. N. Pandya, "Further observations on cortico-frontal connections in the rhesus monkey," Brain Res., vol. 117, pp. 369386, 1976.

[5] E. G. Jones, T. P. S. Powell, An anatomical study of converging sensory pathways within the cerebral cortex of the monkey," Brain, vol. 93, pp. 793-820, 1970. 
[6] J. R. Stroop, "Studies of interference in serial verbal reactions," J. Exp. Psychol. vol. 18, pp. $64362,1935$.

[7] Q. Liang, X. Cheng, S. Huang, D. Chen, "Opportunistic Sensing in Wireless Sensor Networks: Theory and Applications", IEEE Trans on Computers, vol. 63, no. 8, pp. 2002-2010, August 2014.

[8] C. M. MacLeod, "Half a century of research on the Stroop effect: an integrative review," Psychol. Bull., vol. 109, 163203, 1991.

[9] B. Milner, "Effects of different brain lesions on card sorting," Arch. Neurol., vol. 9, pp. 90, 1963.

[10] Q. Liang, X. Cheng, S. Samn, "NEW: Network-enabled Electronic Warfare for Target Recognition," IEEE Trans on Aerospace and Electronic Systems, vol.46, no. 2, pp. 558568, April 2010.

[11] Q. Liang, "Automatic Target Recognition Using Waveform Diversity in Radar Sensor Networks," Pattern Recognition Letters (Elsevier), vol. 29, no. 2, pp. 377-381, 2008.

[12] Q. Liang, "Radar Sensor Wireless Channel Modeling in Foliage Environment: UWB versus Narrowband," IEEE Sensors Journal, vol. 11, no. 6, June 2011, pp. 1448-1457.

[13] S. Singh, et al, "Sense through Wall Human Detection Using UWB Radar," EURASIP Journal on Wireless Communications and Networking, vol. 2011, 2011.

[14] M. Hsu, et al, "Neural systems responding to degrees of uncertainty in human decision making," Science, vol. 310, pp. 1680-1683, Dec 2005. 
[15] Q. Liang, X. Cheng, "KUPS: Knowledge-based Ubiquitous and Persistent Sensor networks for Threat Assessment," IEEE Transactions on Aerospace and Electronic Systems, vol. 44, no. 3, July 2008.

[16] Q. Ren, Q. Liang, "Throughput and energy-efficiency-Aware Protocol for Ultra Wideband Communication in Wireless Sensor Networks: A Cross-Layer Approach," IEEE Transactions on Mobile Computing, vol. 7, no. 7, pp. 805-816, July 2008.

[17] Q. Liang, et al, "TDoA-Based Passive Localization: Terrestrial versus Underwater Environment," IEEE Trans on Parallel and Distributed Processing, vol. 24, no. 10, Oct 2013.

[18] O. Kleinsasser, "Pathogenesis of vocal cord polyps," The Annals of Otology, Rhinology, and Laryngology, vol. 91, pp. 378-81, 1982.

[19] M. de Oliveira Rosa, J. C. Pereira, and M. Grellet, "Adaptive Estimation of Residue Signal for Voice Pathology Diagnosis," IEEE Transactions on Biomedical Engineering, vol. 47 , no. 1,2000 .

[20] J. Jiang, I. Titze "Measurement of vocal fold intraglottal pressure and impact stress," Journal of Voice, vol. 8, no. 2, pp. 132-144, 1994.

[21] Y. Zhang and J. J. Jiang "Chaotic vibrations of a vocal fold model with a unilateral polyp," J. Acoust. Soc. America, vol. 115, no. 3, pp. 1266-1269, March 2004.

[22] L. G. Ceballos, and H. L. Hansen, "Direct Speech Feature Estimation Using an Iterative EM Algoritm for Vocal Fold Pathology Detection," IEEE Trans on Biomedical Engineering, vol. 43, no. 4, April. 1996. 
[23] A. A. Dibazar, S. Narayanan, and T. W. Berger, "Feature Analysis for Automatic Detection of Pathological Speech," Proceedings of the IEEE Engineering in Medicine and Biology Society (EMBS) Meeting, pp. 182-183, 2002.

[24] M. Vasilakis, Y. Stylianou, "Voice Pathology Detection Based on Short-Term Jitter Estimations in Running Speech," Intl J. of Phoniatrics, Speech Therapy and Communication Pathology, vol. 61, no. 3, June 2009.

[25] R. J. Moran, R. B. Reilly, P. de Chazal, and Peter D. Lacy, "Telephony-Based Voice Pathology Assessment Using Automated Speech Analysis," IEEE Trans on Biomedical Engineering, vol. 53, no. 3, pp. 468-477, March 2006.

[26] J. I. Godino-Llorente and P. Gomez-Vilda, "Automatic detection of voice impairments by means of short-term cepstral parameters and neural network- based detectors," IEEE Trans Biomedical Eng., vol. 51, no. 2, pp. 380384, Feb. 2004.

[27] J. M. Mendel, "Uncertainty, fuzzy logic, and signal processing," Signal Processing, vol. 80, no. 6, pp. 913-933, 2000.

[28] Q. Liang and J. M. Mendel, "Interval type-2 fuzzy logic systems: theory and design," IEEE Transactions on Fuzzy Systems, vol. 8, no. 5, pp. 535-550, Oct 2000.

[29] Qilian Liang, Jerry M. Mendel, "Design interval type-2 fuzzy logic systems using SVDQR method: rule reduction", International Journal of Intelligent Systems, vol. 15, no. 10, pp. 939957, Oct 2000 .

[30] L. A. Zadeh, " The concept of a linguistic variable and its application to approximate reasoning - I," Information Sciences, vol. 8, pp. 199-249, 1975.

[31] J. M. Mendel, "Fuzzy logic systems for engineering: a tutorial " Proc. of the IEEE, vol. 83, no. 3, pp. 345-377, March 1995. 
[32] Q. Liang and J. M. Mendel, "Equalization of time-varying nonlinear channels using type-2 fuzzy adaptive filters," IEEE Trans. on Fuzzy Systems, vol. 8, no. 5, pp. 551-563, Oct 2000 .

[33] Q. Liang and J. M. Mendel, " Overcoming time-varying co-channel interference using type-2 fuzzy adaptive filters", IEEE Transactions on Circuits and Systems, II, vol. 47, no. 12, pp. 1419-1428, Dec 2000.

[34] Q. Liang and J. M. Mendel, " MPEG VBR video traffic modeling and classification using fuzzy techniques," IEEE Transactions on Fuzzy Systems, vol. 9, no. 1, pp.183-193, Feb 2001.

[35] Q. Liang, N. Karnik, and J. M. Mendel, "Connection admission control in ATM network using survey-based type-2 fuzzy logic systems," IEEE Transactions on Systems, Man, and Cybernetics, Part C, vol. 30, no. 3, pp. 529-539, August 2000.

[36] H. Shu, et al, "Wireless Sensor Network Lifetime Analysis Using Interval Type-2 Fuzzy Logic Systems," IEEE Transactions on Fuzzy Systems, vol. 16, no. 2, April 2008.

[37] X. Xia and Q. Liang, "Cross-Layer Optimization for Mobile Ad Hoc Networks Using Interval Type-2 Fuzzy Logic Systems," IEEE GLOBECOM, Nov 2006, San Francisco, CA.

[38] X. Xia and Q. Liang, "Cross-Layer Optimization for Mobile Ad Hoc Networks Using Fuzzy Logic Systems," International Journal of Uncertainty, Fuzziness and KnowledgeBased Systems, vol. 16, no. 3, pp. 391-407, June 2008.

[39] Q. Liang, L. Wang, "Event detection in sensor networks using fuzzy logic system," IEEE Intl Conference on Computational Intelligence for Homeland Security and Personal Safety, March 2005, Orlando, FL. 
[40] Q. Ren, Q. Liang, Fuzzy logic-optimized secure media access control (FSMAC) protocol for wireless sensor networks," IEEE Intl Conference on Computational Intelligence for Homeland Security and Personal Safety, March 2005, Orlando, FL.

[41] L. R. Rabiner, "A tutorial on hidden Markov models and selected applications in speech recognition," Proceedings of the IEEE, vol. 77, no. 2, pp. 257286, February 1989

[42] K. Tanaka, M. Sano, and H. Watanabe, "Modeling and control of carbon monoxide concentration using a neuro-fuzzy technique" IEEE Trans Fuzzy Systems, vol. 3, no. 3, pp. 271-279, Aug 1995.

[43] R. O. Duda and P. E. Hart, Pattern Classification and Scene Analysis, John Wiley \& Sons, Inc, USA, 1973.

[44] Z. Zhong, et al, "Vocal Folds Damage Detection Based on Patient Voices," Lecture Notes in Electrical Engineering vol. 202, pp. 531 539, 2012.

[45] W. Wang, et al, "Throat polyp detection based on compressed big data of voice with support vector machine algorithm," EURASIP Journal on Advances in Signal Processing, vol. 2014 .

[46] W. Wang, et al, "Intelligent throat polyp detection with separable compressive sensing," EURASIP Journal on Advances in Signal Processing, vol. 2014. 
Table 1: An example: mean and std values for 10 segments (10,000 samples/segment) and the entire voice (100,000 samples) from one person, and their normalized std.

\begin{tabular}{|l||c|c|c|c|}
\hline \multirow{2}{*}{ Voice Data } & \multicolumn{2}{|c|}{ Vowel /a:/ } & \multicolumn{2}{c|}{ Vowel $/ \mathrm{i} /$} \\
\cline { 2 - 5 } & mean & std & mean & std \\
\hline \hline Segment 1 & -9.6012 & $9.8983 \times 10^{4}$ & 616.1160 & $3.9892 \times 10^{4}$ \\
\hline Segment 2 & 2.2539 & $9.7968 \times 10^{4}$ & -551.6035 & $4.9503 \times 10^{4}$ \\
\hline Segment 3 & -6.4511 & $9.8753 \times 10^{4}$ & -26.1122 & $4.7019 \times 10^{4}$ \\
\hline Segment 4 & -0.8452 & $9.7641 \times 10^{4}$ & -204.7233 & $4.5823 \times 10^{4}$ \\
\hline Segment 5 & 14.5417 & $9.6117 \times 10^{4}$ & 801.8690 & $4.2358 \times 10^{4}$ \\
\hline Segment 6 & -14.2853 & $9.5306 \times 10^{4}$ & -762.8290 & $4.1863 \times 10^{4}$ \\
\hline Segment 7 & 0.9474 & $9.7104 \times 10^{4}$ & -20.7100 & $4.5593 \times 10^{4}$ \\
\hline Segment 8 & 7.6797 & $9.7449 \times 10^{4}$ & -37.0950 & $4.6036 \times 10^{4}$ \\
\hline Segment 9 & 10.6494 & $9.7594 \times 10^{4}$ & 522.1380 & $4.6445 \times 10^{4}$ \\
\hline Segment 10 & -15.1029 & $9.7751 \times 10^{4}$ & -50.2020 & $4.2348 \times 10^{4}$ \\
\hline Entire Voice & -1.0214 & $9.7468 \times 10^{4}$ & 29.8584 & $4.4774 \times 10^{4}$ \\
\hline Normalized std & 10.0384 & 0.0113 & 16.6340 & 0.0655 \\
\hline
\end{tabular}


Table 2: The classification performance and number of parameters in 4 different classifiers.

\begin{tabular}{|l||c|c|}
\hline Classifier & $p_{r}(\epsilon)$ & Total Number of Parameters \\
\hline Type-1 Fuzzy Classifier & $18 \%$ & 80 \\
\hline Type-2 Fuzzy Classifier & $14 \%$ & 120 \\
\hline Bayesian Classifier & $25 \%$ & 80 \\
\hline Linear Classifier & $21 \%$ & 45 \\
\hline
\end{tabular}




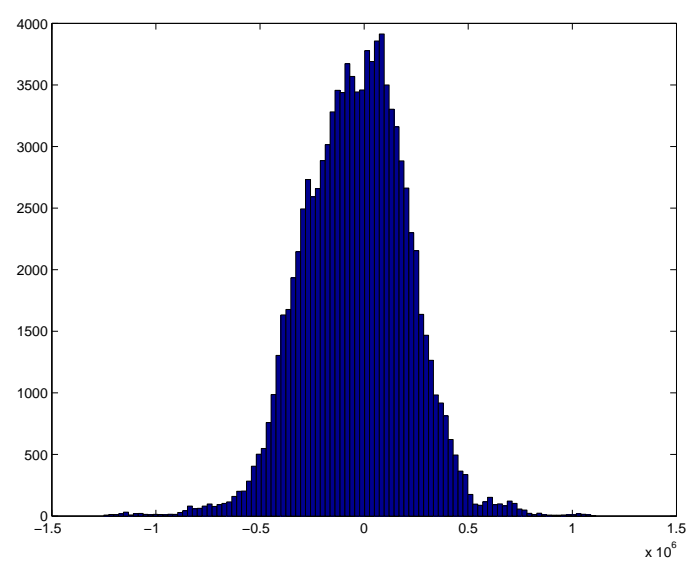

(a)

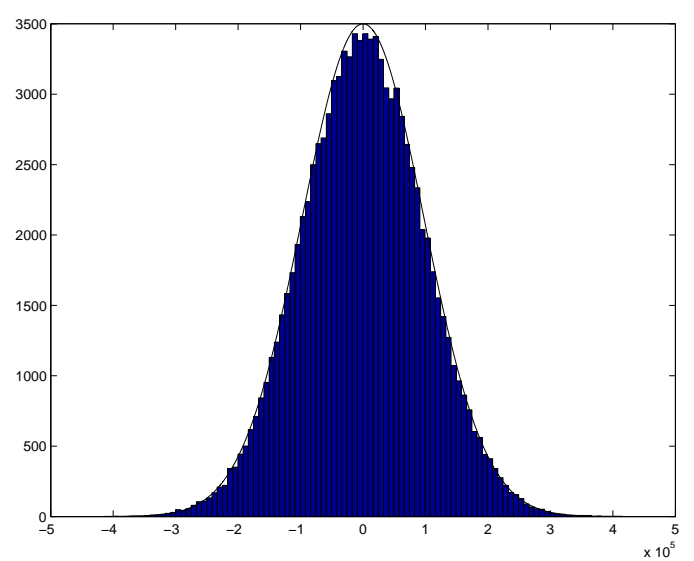

(b)

Figure 1: (a) The histogram of 100,000 voice /a:/ samples $x_{k}$; (b) the histogram of transformed voice samples $x_{k}-x_{k-5}$ and its matching to a Gaussian distribution. 


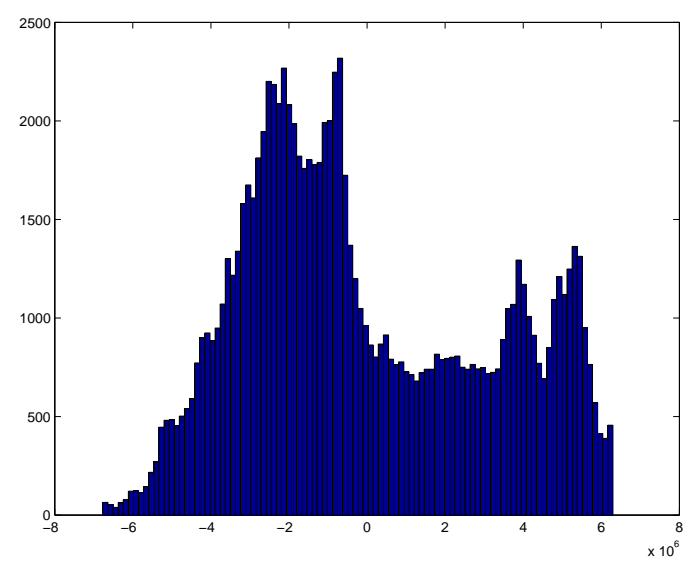

(a)

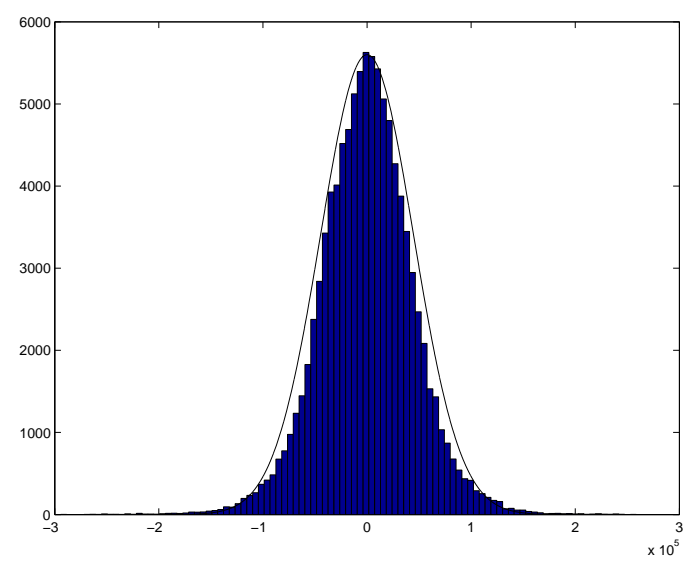

(b)

Figure 2: (a) The histogram of 100,000 voice /i:/ samples $x_{k}$; (b) the histogram of transformed voice samples $x_{k}-x_{k-1}$ and its matching to a Gaussian distribution. 


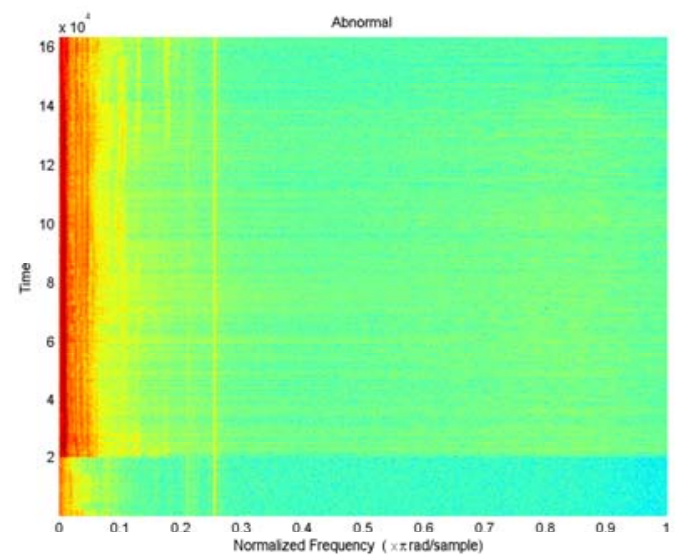

(a)

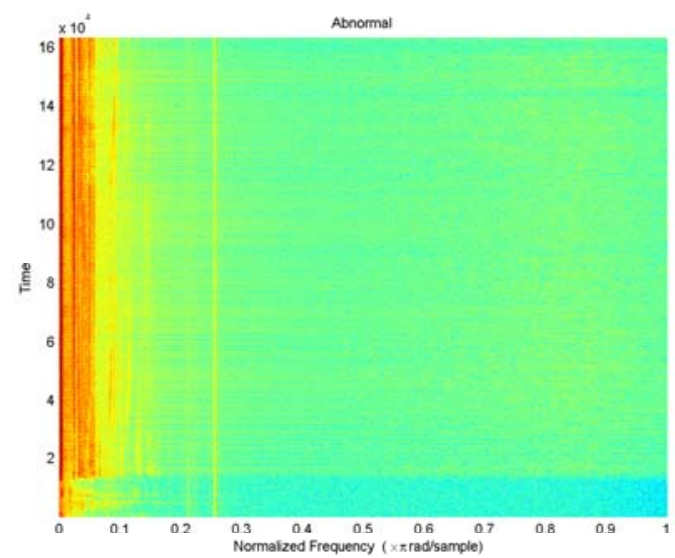

(c)

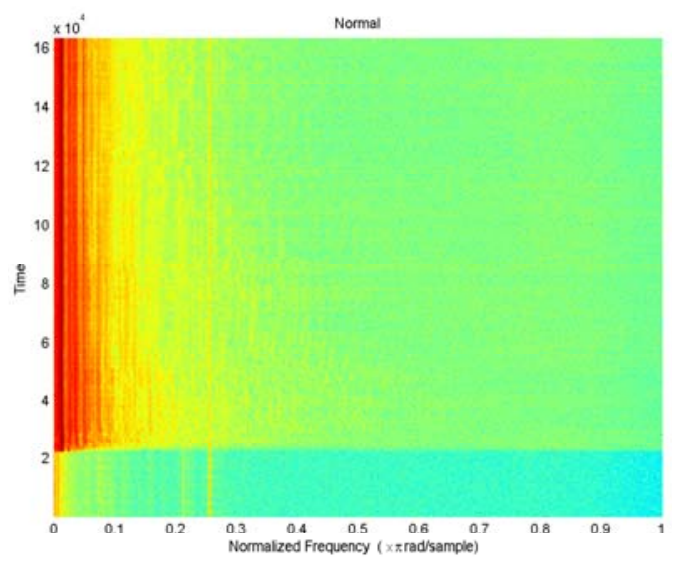

(b)

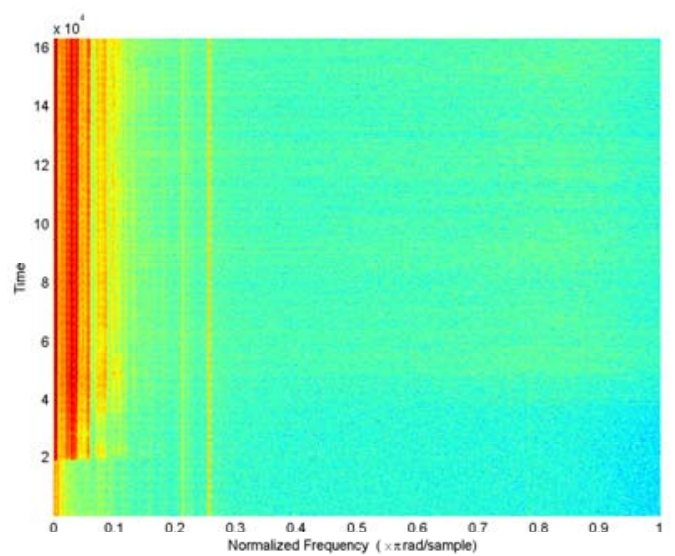

(d)

Figure 3: Spectrogram using a Short-Time Fourier Transform (STFT). Window size of STFT is 2048, and overlap between two neighbor window is 1024. (a) is from a vocal folds damage patient speaking vowel /a:/; (b) is from a normal person speaking vowel /a:/; (c) is from the vocal folds damage patient speaking vowel /i:/; (d) is from the normal person speaking vowel /i:/. 


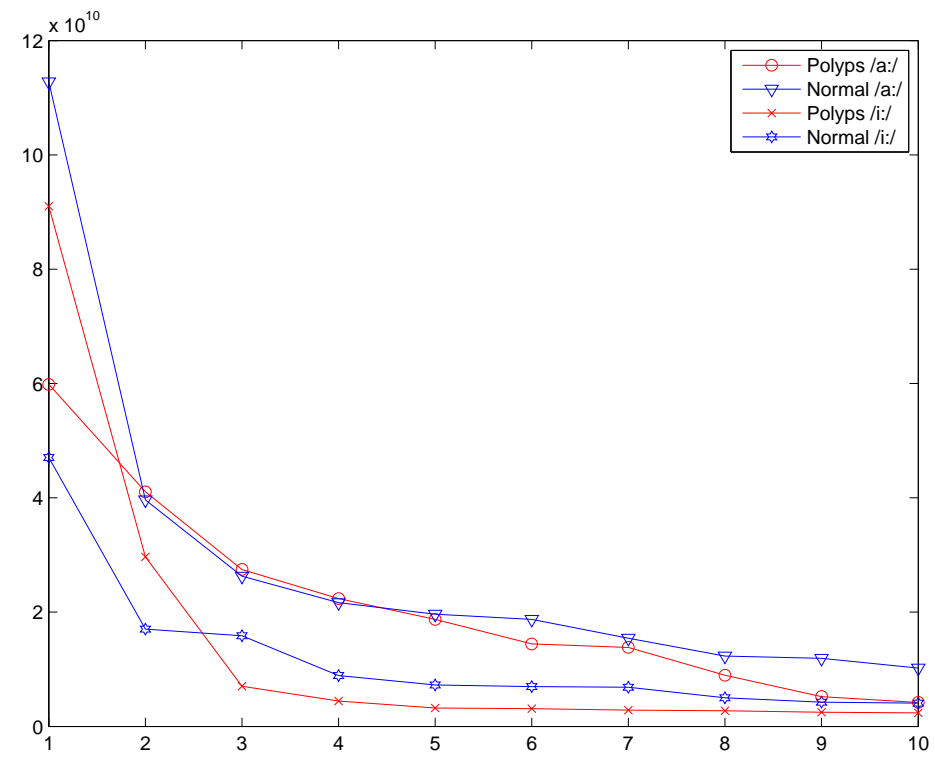

Figure 4: The 10 largest singular values of STFT of two patients in Fig. 3. 